\title{
Association of tumour-associated macrophages with cancer cell EMT, invasion, and metastasis of Kazakh oesophageal squamous cell cancer
}

Jihong Liu ${ }^{1,3+}$, Chunxiao Li ${ }^{1 \dagger}$, Liyan Zhang ${ }^{4 \dagger}$, Kai Liu', Xianli Jiang ${ }^{1}$, Xueli Wang ${ }^{1}$, Lan Yang ${ }^{1}$, Weihua Liang ${ }^{1}$, Kunping $\mathrm{Liu}^{3}$, Jianming $\mathrm{Hu}^{1 *}$ and Feng $\mathrm{Li}^{1,2^{*}}$

\begin{abstract}
Background: Tumour-associated macrophages (TAMs) play an important role in the growth, progression, and metastasis of tumours. Epithelial-mesenchymal transition (EMT) is a mechanism for tumour invasion and metastasis. In this study, we aimed to determine whether TAMs can induce EMT for the invasion and metastasis of Kazakh oesophageal squamous cell cancer (ESCC).

Methods: CD163 was used as a marker for TAMs, and the density of TAMs in tumour nest and surrounding stroma was quantified using immunohistochemistry (IHC). IHC staining was used to evaluate the expression of E-cadherin (epithelial marker) and vimentin (mesenchymal marker) in Kazakh ESCC and cancer-adjacent normal tissues (CANs). Additionally, 6-well transwell plates $(0.4 \mu \mathrm{m})$ were used to establish the co-culture system of ESCC (EC109 or EC9706) cells and macrophages. Real-time quantitative polymerase chain reaction (qPCR) and western blot experiments were used to determine whether ESCC cells undergo EMT transformation after co-culture with macrophages. Transwell assays were used to detect the migration and invasion of the ESCC cells.

Results: The distribution of CD163-positive TAMs in cancer tissues was closely related to EMT in Kazakh ESCC. The expression of vimentin in the ESCC cells was significantly upregulated, the expression of E-cadherin was significantly downregulated, and the invasion and migration of the ESCC cells were significantly enhanced after tumourassociated macrophages were added to the co-culture.
\end{abstract}

Conclusions: Tumour-associated macrophages promote EMT in ESCC, which may be one of the important factors involved in the invasion and progression of Kazakh ESCC.

Keywords: Oesophageal squamous cell carcinoma, Tumour-associated macrophages, Kazakh, Epithelialmesenchymal transition, Migration and invasion

\section{Background}

Oesophageal cancer (EC) is the eighth most common malignancy in the world and is the sixth most common cause of cancer deaths [1]. The incidence and mortality rates of Kazakh EC in northwest Xinjiang are higher than the average levels in China [2]. Early invasion and

\footnotetext{
*Correspondence: jianming.120@163.com; lifeng7855@126.com

Jihong Liu, Chunxiao Li and Liyan Zhang are Co-first authors.

'Department of Anatomic and Surgical Pathology, and Key Laboratory of Xinjiang Endemic and Ethnic Diseses, The First Affiliated Hospital, Shihezi University School of Medicine, Xinjiang 832002, People's Republic of China Full list of author information is available at the end of the article
}

metastasis may be the main reasons for poor prognosis of Kazakh EC.

There is growing evidence that the tumour microenvironment is important for tumour progression and metastasis [3-5]. Macrophages are important mesenchymal cells in the tumour microenvironment that play an important role in promoting tumour progression, angiogenesis, and metastasis [6]. Macrophages possess plasticity and are classified into different subtypes in different environments, namely, M1 classically activated macrophages, and M2 alternatively activated macrophages [6].

(c) The Author(s). 2019 Open Access This article is distributed under the terms of the Creative Commons Attribution 4.0 International License (http://creativecommons.org/licenses/by/4.0/), which permits unrestricted use, distribution, and 
Macrophages can differentiate into M1 macrophages in the presence of lipopolysaccharide (LPS) and interferon gamma (IFN $\gamma$ ), release a series of cytokines, result in the low expression of interleukin (IL-10) and high expression of IL-12, and inhibit and kill cancer cells. Macrophages can differentiate into the M2 subtype in the presence of IL-4 and IL-13, low expression of IL-12, and high expression of IL-10, which plays an important role in inhibiting inflammation and promoting tumour growth and immunosuppression [7]. Macrophages present in tumours (tumour-associated macrophages or TAMs) exhibit M2 phenotype and are capable of expressing CD68 and CD163 [8]. Recent studies have shown that CD163-positive macrophages are associated with histological grade and poor prognosis of tumours [9]. TAM infiltration density is associated with recurrent and poor prognosis in a variety of tumours such as breast cancer [10], leiomyosarcoma [11], and oesophageal cancer [6]. However, the underlying mechanisms of TAMs in the invasion and metastasis of EC are unclear.

Currently, epithelial-mesenchymal transition (EMT) is considered a mechanism of tumour invasion and metastasis [12]. EMT refers to the conversion of epithelial cells into mesenchymal cells under certain conditions [13]. The cells are rounded into a spindle shape and become motile and invasive [14]. A series of evidence suggests that TAMs can promote invasion and metastasis of breast cancer [15], lung cancer [16], and hepatocellular carcinoma [17] through EMT. However, the role of EMT in TAM-promoted invasion and metastasis of ESCC is not yet known.

Therefore, CD163 was used as a marker of M2 TAMs, E-cadherin and vimentin were used as epithelial and mesenchymal markers, respectively, and the co-culture system was established, to explore whether TAMs could induce EMT for the invasion and metastasis of Kazakh ESCCs.

\section{Methods}

\section{Patients and specimens}

Surgically resected and paraffin-embedded human tissues were collected from the Department of Pathology at Yili Friendship Hospital in Xinjiang, China, between 2008 and 2014. One hundred cases of Kazakh ESCC and 100 cases of Kazakh cancer-adjacent normal (CAN) tissues were collected for this study(some of cases overlaps with previous study [18]). Of all patients, 62 were males and $38 \mathrm{fe}$ males, with ages ranging from 33 to 76 years and a mean age of 58 years. All patients were diagnosed with ESCC were confirmed by two pathologists according to the World Health Organization histological tumour classification criteria. In this study, there were 26 patients with well differentiated, 47 patients with moderately differentiated, and 27 patients with poorly differentiated ESCCs. From these, 29 and 71 patients exhibited invasive depths of T1T2 and T3-T4, respectively. There were 45 patients with lymph node metastasis, 68 patients with lymphatic metastasis, 65 patients in clinical stages I-II, and 35 patients in clinical stages III-IV. None of the patients accepted chemotherapy or radiation therapy before surgery.

\section{Immunohistochemistry (IHC)}

For IHC, anti-CD163 (clone 10D6, Zhongshan Goldenbridge Biotechnology Co., LTD., Beijing, China) was diluted 1:100. Both anti-E-cadherin and anti-vimentin antibodies were purchased from Cell Signaling Technology (Danvers, MA, USA) and diluted 1:1000. The sections were thoroughly washed with PBS and the primary antibody binding was visualized using the DAKO Envision kit (DAKO, Glostrup, Denmark) according to the manufacturer's instructions. IHC was performed as reported previously [19].

\section{Immunoreactivity evaluation}

CD163-positive macrophages were analysed as described previously [19]. Tumour nest and stroma areas were defined, and CD163-positive macrophages were counted under high-power fields (HPFs) $(400 \times)$ by two pathologists who were blinded to the clinical patient data. When cell counts differed by more than 10 cells per HPF, the cells would be counted again a week later until the recorded differences were below 10 counts. The mean number of macrophages per HPF across five hot spots for every sample (tumour islet and tumour stroma) was defined as the M2 macrophage density.

E-cadherin and vimentin were positively expressed on the membrane or cytoplasm of the cell, which were defined as a yellow-brown colour, following the manufacturer's guidelines. IHC-stained slides were scored as positive or negative by the percentage and intensity of positive cells. The scoring percentages of positively stained cells were as follows: $0,<5 \% ; 1,6-25 \% ; 2,26-50 \%$; 3, 51$75 \%$; and $4,76-100 \%$. The staining intensity scoring was as follows: 0 , absent; 1 , weak; 2 , moderate; and 3 , strong. A final score was based on the multiplication of both scores from individual slides [19], where $0-1$ was negative $(-), 2-3$ was weakly positive $(1+), 4-6$ was moderately positive $(2+)$, and $8-12$ was strongly positive $(3+)$.

\section{Cell culture}

Human ESCC cell lines EC109 and EC9706 and the human acute monocytic leukaemia cell line THP-1 were purchased from the American Type Culture Collection (ATCC; Manassas, VA, USA). These cells were cultured in RPMI-1640 medium (Invitrogen, Carlsbad, CA, USA) containing 10\% foetal bovine serum (FBS; Invitrogen, Carlsbad, CA, USA) and $1 \%$ penicillin/streptomycin solution (Invitrogen, Carlsbad, CA, USA). THP-1 cells were additionally incubated with $0.05 \mathrm{mM} \beta$-mercaptoethanol. All cells were incubated at $37^{\circ} \mathrm{C}$ in a humidified cell culture incubator with $5 \% \mathrm{CO}_{2}$. THP-1 cells were treated with Phorbol-12-myristate-13-acetate (PMA, $10 \mathrm{ng} / \mathrm{mL}$; Sigma, USA) for $36 \mathrm{~h}$ to induce THP-1 
to macrophages, then macrophages and EC109 or EC9706 cells $\left(4 \times 10^{4}\right.$ cells) were separately cultured in the upper and lower chamber of 6-well transwell plates (Corning Inc., Corning, NY) for $48 \mathrm{~h}$ without contact, the supernatant was collected and filtered, which was co-cultured conditioned medium (CM). At the same time, ESCC cells cultured in the DMEM for $48 \mathrm{~h}$, the supernatant was collected and filtered, which was routine medium (RM) as control. Finally, the cocultured conditioned medium (CM) and RM was collected for subsequent studies.

\section{Quantitative polymerase chain reaction (qPCR)}

Total RNA was extracted using TRIzol reagent (Invitrogen) according to the manufacturer's instructions, and mRNA was reverse-transcribed into cDNA by using the TIANScript RT Kit (TIANGEN, China) at $37^{\circ} \mathrm{C}$ for 15 min and $85^{\circ} \mathrm{C}$ for $5 \mathrm{~s}$, according to the manufacturer's protocol. qPCR was performed to evaluate the gene expression using QuantiFast SYBR Green PCR (QIAGEN) with a 7500 fast Real-Time PCR System (Applied Biosystems, Foster City, CA, USA) under the following conditions: 40 cycles at $95^{\circ} \mathrm{C}$ for $30 \mathrm{~s}, 95^{\circ} \mathrm{C}$ for $5 \mathrm{~s}$, and $60^{\circ} \mathrm{C}$ for $31 \mathrm{~s}$. The primers used were previously published [20].

\section{Western blotting}

Cells were washed 3 times with ice-cold phosphate buffer saline (PBS) and were lysed by lysis buffer on ice for $30 \mathrm{~min}$, followed by centrifugation at $12,000 \mathrm{rpm}$ for 10 min at $4{ }^{\circ} \mathrm{C}$ to remove cell debris. Protein samples were mixed with loading buffer, heated at $100^{\circ} \mathrm{C}$ for $10 \mathrm{~min}$, and separated on $10 \%$ sodium dodecyl sulphate- polyacrylamide gel electrophoresis (SDS-PAGE) gels (BioRad, California, USA). Proteins were then transferred to polyvinyl difluoride membranes (Solarbio Systems, Beijing, China). The blots were incubated with primary antibodies overnight at $4{ }^{\circ} \mathrm{C}$ with rabbit anti-E-cadherin (1:400) (Cell Signaling Technology, Danvers, MA, USA) and rabbit anti-vimentin (1:400) (Cell Signaling Technology, Danvers, MA, USA). This procedure was followed by incubation with an anti-rabbit HRP-labelled secondary antibody $(1: 20,000)$ for $2 \mathrm{~h}$ at $25^{\circ} \mathrm{C}$, followed by detection with an Enhanced-Chemi-Luminescence (ECL) reagent kit (Thermo Systems, Massachusetts, USA). Immunoblots for $\beta$-actin served as a loading control. All experiments were repeated in triplicate.

\section{Cell migration and invasion assays}

Cells $\left(4 \times 10^{4}\right)$ were diluted in $200 \mu \mathrm{L}$ serum-free medium and then added to the top chambers of 24-well plates (Corning, Corning, NY, USA); $600 \mu \mathrm{L}$ of $20 \%$ FBS was added to the lower chamber to induce cell migration. Each chamber was coated freshly with $50 \mu \mathrm{g}$ Matrigel (Becton, Dickinson and Company, Franklin Lakes, NJ, USA) before performing the invasion assay. After 24 $\mathrm{h}$, the cells were fixed with $4 \%$ paraformaldehyde and then stained with $0.1 \%$ crystal violet. The number of migrated and invasive cells was calculated in five random fields per well using an inverted light microscope.

\section{Statistical analysis}

Data were analysed using the SPSS 17.0 software package. Two sets of measurement data were compared using Independent-Samples $t$ test and $\chi^{2}$ test. Comparisons between multiple groups were performed using Partial correlation analysis. $P$ values less than 0.05 were considered statistically significant. $\mathrm{P}$ values were corrected with Bonferroni Correction for multiple testing.

\section{Results}

\section{Distribution of TAMs in Kazakh ESCC}

In this study, we used CD163 as a marker of TAMs to assess their distribution in the Kazakh ESCC patients. CD163 was localized in the cell membrane or in the cytoplasm of macrophages. The TAMs mainly localized in the stroma of cancerous tissues, and a few localized in the cancerous nest (Fig. 1). The number of TAMs in ESCC was significantly higher in the interstitial tissues (58 per $\mathrm{HPF}$ ) and in the nest (13 per HPF) than in the CAN stroma (22 per HPF) and epithelium (2 per HPF), and the difference was statistically significant $(p<0.05)$ (Table 1$)$.

\section{Expression of EMT-related proteins in Kazakh ESCC and their correlation with clinicopathological parameters}

We used E-cadherin and vimentin as markers for the epithelium and mesenchyme, respectively, to detect EMT in Kazakh ESCC. We found that E-cadherin predominantly localized in the cell membrane and vimentin localized in the cell membrane or cytoplasm (Fig. 2). The positive expression of E-cadherin in ESCC tumour tissues was 58\% (58/100), which was significantly lower than that in CANs tissues $(96 \%, p<0.05)$ (Table 2). While the expression of vimentin in ESCC tumour tissues was 47\% (47/100), and none of the normal tissues expressed vimentin in CAN tissues (0\%), (Table 2). We further examined the correlation of EMTrelated protein expression and the clinicopathological characteristics as shown in Table 3. Despite no significant correlations with sex and age, we observed a significant negative correlation between E-cadherin expression and tumour differentiation, clinical stage, depth of invasion, lymph node metastasis, and vascular invasion $(p<0.05)$, Moreover, we found that the expression of E-cadherin was negatively correlated with tumour differentiation, especially between moderate differentiation and poor differentiation $(p<0.0125)$. However, we found that the expression of vimentin was positively correlated with gender, depth of invasion, clinical stage, lymph node metastasis, and vascular invasion $(p<0.05)$. No positive correlations were found between other clinicopathological parameters and vimentin expression. 


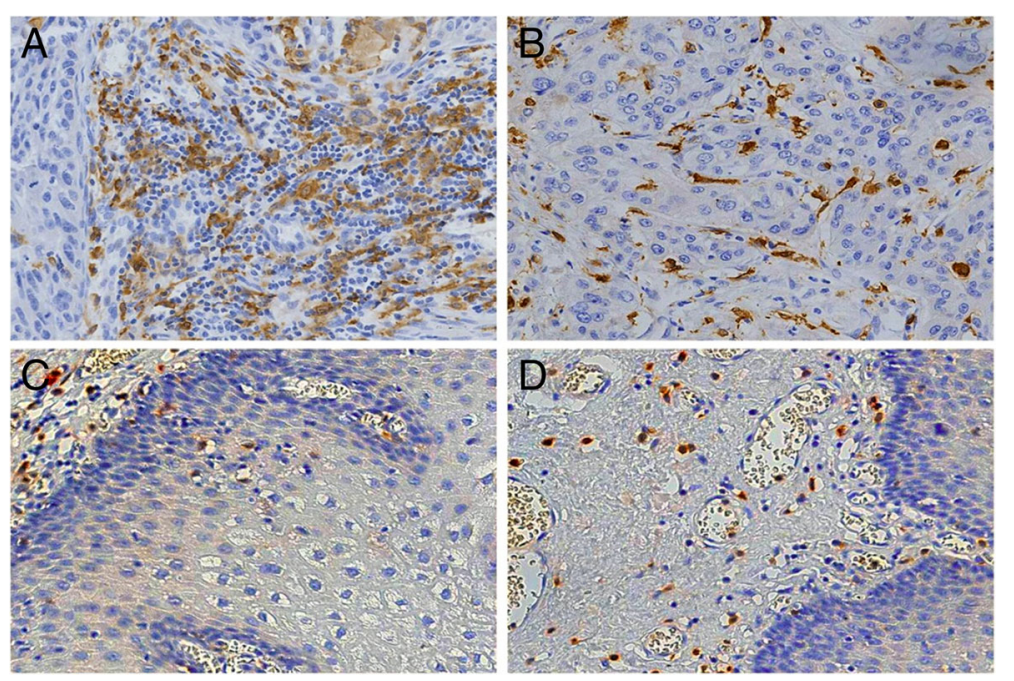

Fig. 1 Distribution of CD163-positive M2 macrophages in Kazakh ESCC and CAN tissues. (a) and (b) showed the distribution of M2 macrophages in ESCC tumour stromal and islet, respectively. CD163 revealed diffuse staining of membranes and cytoplasm of M2 macrophages and showed the high density of M2 macrophages located in ESCC tissues (especially in tumour stroma). (c) and (d) showed the distribution of TAMs in CAN stroma and epithelia, respectively. A small number of CD163-positive M2 macrophages appear in CAN tissues

\section{Distribution of TAMs was associated with EMT in Kazakh ESCC tissues}

To investigate the role of TAMs in the tumour EMT process, the relationship between the distribution of TAMs and EMT-related protein was analysed. The density of interstitial TAM infiltrates positively correlated with vimentin expression $(p<0.05)$ and was not related with Ecadherin expression $(p>0.05)$. There was a negative correlation between the expression of E-cadherin and vimentin $(p<0.05)$ (Table 4$)$. These results indicated that TAMs were positively associated with EMT in ESCC.

\section{TAMs induce cancer cells to undergo EMT in ESCC}

THP-1 cells are usually selected as a model for studying human macrophage differentiation. In this study, suspended, round-shaped THP-1 cells were induced to adherent macrophages upon treatment with PMA for $36 \mathrm{~h}$ (Fig. 3a). To investigate whether TAMs could promote EMT of ESCC cells, we co-cultured THP-1-derived macrophages with ESCC cell lines (EC109 and EC9706) for 48 $h$ and found that the morphology of ESCC cells transformed from round epithelial cells into loose, scattered, spindle-shaped mesenchymal cells, suggesting that EMT occurred in the presence of macrophages (Fig. 3b). To further confirm EMT, the pattern of gene expression was

Table 1 The distribution of CD163-positive macrophages in Kazakh ESCCs and CANs

\begin{tabular}{llllll}
\hline Groups & Cases $(\mathrm{N})$ & $\begin{array}{l}\text { Islets } \\
(\text { mean } \pm \text { SD) }\end{array}$ & $P$ & $\begin{array}{l}\text { Stroma } \\
(\text { mean } \pm \text { SD })\end{array}$ & $P$ \\
\hline ESCCS & 100 & $13.43 \pm 9.22$ & $<0.001$ & $57.96 \pm 26.53$ & $<0.001$ \\
CANs & 100 & $1.82 \pm 1.71$ & & $22.23 \pm 9.85$ & \\
\hline
\end{tabular}

analysed by qPCR. mRNA expression of E-cadherin decreased, whereas that of vimentin increased in EC109 and EC9706 cells $(p<0.05)$ (Fig. 3c). Western blot analysis showed that the expression of E-cadherin decreased significantly, whereas that of vimentin increased significantly in the presence of TAMs $(p<0.05)$ (Fig. 3d). These results suggest that TAMs promoted EMT of ESCC cells, which were consistent with the results from in vivo tissue studies.

TAMs enhance cancer cell migration and invasion in vitro A transwell assay was used to investigate whether TAMs also influenced the migration and invasion ability of ESCC cells. The results in Fig. $3 \mathrm{e}$ and $\mathrm{f}$ showed that the migration and invasion ability of ESCC cells cultured in macrophage CM was significantly higher than that of cancer cells cultured in regular media $(p<0.05)$, suggesting that TAMs promoted the migration and invasion of ESCC.

\section{Discussion}

TAMs are involved in tumour progression and are associated with poor prognosis in several cancers [21-24]. To our knowledge, EMT is one of the most important mechanisms of tumour invasion and metastasis [25]. However, the interaction between the TAMs and EMT in ESCC has not been elucidated clearly. Investigation of the underlying mechanisms may reveal new targets for EC treatment.

Our previous study shows that the number of M2 TAMs positively correlated with malignant phenotypes including lymph node metastasis and clinical stage progression of Kazakh ESCC [18]. On the basis of previous studies, we add some new Kazakh ESCC samples and found that CD163-positive TAMs were primarily located in the 


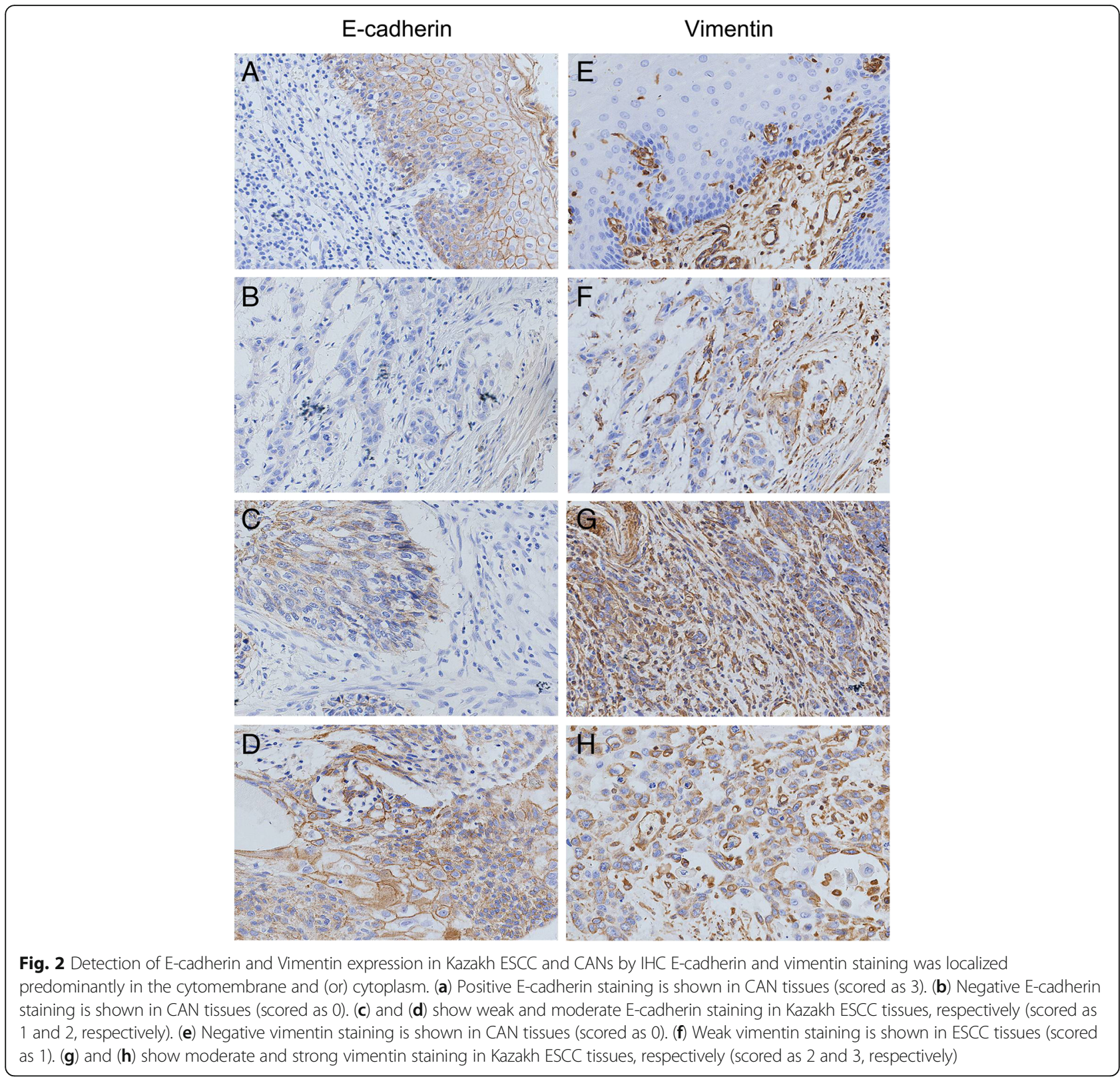

tumour stroma, and a few were also distributed in the tumour islet. The density of TAMs in the tumour islet and stroma was significantly higher than that in the corresponding CAN tissues. Our results were similar to those reported in gastric cancer [26], oral carcinoma [27], and endometrial adenocarcinoma [28], indicating that TAMs play a crucial role in the promotion of oesophageal cancer invasion and metastasis. EMT is required for tumour invasion and metastasis. To investigate whether TAMs were involved in EMT of cancer cells to promote the invasion and metastasis of ESCCs, we analysed the expression of an epithelial marker (E-cadherin) and mesenchymal marker

Table 2 The expression of E-cadherin and Vimentin in Kazakh esophageal ESCC CANs

\begin{tabular}{|c|c|c|c|c|c|c|c|c|c|}
\hline \multirow[t]{3}{*}{ Groups } & \multirow[t]{3}{*}{ Cases } & \multicolumn{2}{|c|}{ E-cadherin expression } & \multirow[t]{3}{*}{$x^{2}$} & \multirow[t]{3}{*}{$P$} & \multicolumn{2}{|c|}{ Vimentin expression } & \multirow[t]{3}{*}{$x^{2}$} & \multirow[t]{3}{*}{$P$} \\
\hline & & Low & high & & & low & high & & \\
\hline & & $0 / 1+(\%)$ & $2+/ 3+(\%)$ & & & $0 / 1+(\%)$ & $2+/ 3+(\%)$ & & \\
\hline ESCCS & 100 & $42(42 \%)$ & $58(58 \%)$ & 40.768 & $<0.001$ & $53(53 \%)$ & $47(47 \%)$ & 61.438 & $<0.001$ \\
\hline CANs & 100 & $4(4 \%)$ & $96(96 \%)$ & & & $100(100 \%)$ & $0(0 \%)$ & & \\
\hline
\end{tabular}


Table 3 Correlation between expression of EMT and clinicopathological parameters in Kazakh ESCCs

\begin{tabular}{|c|c|c|c|c|c|c|c|c|c|}
\hline Variable & Cases & $\begin{array}{l}\text { E-cadherin low } \\
\text { expression } 0 / 1+(\%)\end{array}$ & $\begin{array}{l}\text { E-cadherin high } \\
\text { expression } 2+/ 3+(\%)\end{array}$ & $x^{2}$ & $P$ & $\begin{array}{l}\text { Vimentin low } \\
\text { expression } 0 / 1+(\%)\end{array}$ & $\begin{array}{l}\text { Vimentin high } \\
\text { expression } 2+/ 3+(\%)\end{array}$ & $x^{2}$ & $P$ \\
\hline \multicolumn{10}{|l|}{ Age(y) } \\
\hline$\leq 58$ & 58 & $29(50 \%)$ & $29(50 \%)$ & 3.628 & 0.057 & $30(51.7 \%)$ & $28(48.3 \%)$ & 0.090 & 0.764 \\
\hline$>59$ & 42 & 13(31\%) & $29(69 \%)$ & & & $23(54.8 \%)$ & $19(45.2 \%)$ & & \\
\hline \multicolumn{10}{|l|}{ Gender } \\
\hline Male & 62 & $30(48.4 \%)$ & $32(51.6 \%)$ & 2.732 & 0.098 & $26(41.9 \%)$ & $36(58.1 \%)$ & 8.109 & $0.005^{*}$ \\
\hline Female & 38 & $12(31.6 \%)$ & $26(68.4 \%)$ & & & $27(71.1 \%)$ & $11(28.9 \%)$ & & \\
\hline \multicolumn{10}{|c|}{ Differentiation } \\
\hline High & 26 & $9(34.6 \%)$ & $17(65.4 \%)$ & $0.055^{\mathrm{a}}$ & $0.814^{\mathrm{a}}$ & 13(50\%) & 13(50\%) & & \\
\hline Moderate & 47 & $15(31.9 \%)$ & $32(68.1 \%)$ & $8.382^{b}$ & $0.004^{\mathrm{b \#}}$ & $30(63.8 \%)$ & $17(36.2 \%)$ & 5.069 & 0.079 \\
\hline Poor & 27 & 18(66.7\%) & $9(33.3 \%)$ & $5.445^{c}$ & $0.020^{c}$ & $1(37 \%)$ & $2(63 \%)$ & & \\
\hline \multicolumn{10}{|c|}{ Depth of invasion } \\
\hline T1-T2 & 29 & $3(10.3 \%)$ & $26(89.7 \%)$ & 16.802 & $<0.001^{*}$ & $25(86.2 \%)$ & $4(13.8 \%)$ & 18.081 & $\begin{array}{l}< \\
0.001^{*}\end{array}$ \\
\hline T3-T4 & 71 & $39(54.9 \%)$ & $32(45.1 \%)$ & & & 28(39.4\%) & $43(60.6 \%)$ & & \\
\hline \multicolumn{10}{|c|}{ Clinical stage } \\
\hline$|-| \mid$ & 65 & 19(29.2\%) & $46(70.8 \%)$ & 12.431 & $<0.001^{*}$ & $45(69.2 \%)$ & $20(30.8 \%)$ & 19.640 & $\begin{array}{l}< \\
0.001^{*}\end{array}$ \\
\hline III-IV & 35 & $23(65.7 \%)$ & $12(34.3 \%)$ & & & $8(22.9 \%)$ & $27(77.1 \%)$ & & \\
\hline \multicolumn{10}{|c|}{ Lymph node metastasis } \\
\hline pN- & 55 & $18(32.7 \%)$ & $37(67.3 \%)$ & 4.314 & $0.038^{*}$ & $37(67.3 \%)$ & $18(32.7 \%)$ & 9.995 & $0.002^{*}$ \\
\hline $\mathrm{pN}+$ & 45 & $24(53.3 \%)$ & $21(46.7 \%)$ & & & $16(35.6 \%)$ & $29(64.4 \%)$ & & \\
\hline \multicolumn{10}{|c|}{ Lymphatic metastasis } \\
\hline $\mathrm{pN}-$ & 32 & $7(21.9 \%)$ & $25(78.1 \%)$ & 7.824 & $0.005^{*}$ & $23(71.9 \%)$ & $9(28.1 \%)$ & 6.730 & $0.009^{*}$ \\
\hline $\mathrm{pN}+$ & 68 & $35(51.5 \%)$ & $33(48.5 \%)$ & & & $30(44.1 \%)$ & $43(55.9 \%)$ & & \\
\hline
\end{tabular}

${ }^{*} p<0.05 .{ }^{*} p<0.0125$

a compared to moderate differentiation

b compared to poor differentiation

c compared to high differentiation

(vimentin) in ESCC samples. We found that E-cadherin expression negatively correlated with the number of TAMs and was significantly downregulated in ESCC. However, vimentin expression positively correlated with number of TAMs and was significantly upregulated in ESCC. Furthermore, we found that the expression of E-cadherin was significantly down-regulated in ESCCs compared with CAN tissues. Low expression of E-cadherin was closely related to the depth of invasion, clinical stage, lymph node metastasis and vascular invasion of ESCCs. Whereas the expression of Vimentin was significantly up-regulated in ESCCs, and the overexpression of Vimentin was closely related to the depth of invasion, clinical stage, lymph node metastasis and vascular invasion of ESCCs. Through the analysis of Partail correlation, found that M2 TAMs in tumour stroma were positively correlated with Vimentin, but negatively correlated with E-cadherin in ESCCs, suggesting that the cancer cells may be reprogrammed by the

Table 4 Cross correlation analyses reveal strong relationships among density of TAM in tumor nest, tumor stroma and expression of E-cadherin and Vimentin in Kazakh ESCCS

\begin{tabular}{llll}
\hline Characteristics & $\begin{array}{l}\text { TAM density in tumor nest } \\
r / P\end{array}$ & $\begin{array}{l}\text { TAM density in tumor stroma } \\
r / P\end{array}$ & $\begin{array}{l}\text { E-cadherin } \\
r / P\end{array}$ \\
\hline TAM density in tumor nest & 1 & 1 & 1 \\
TAM density in tumor stroma & $0.380 /<0.001^{*}$ & $-0.112 / 0.272$ & $-0.619 /<0.001^{*}$ \\
E-cadherin & $0.066 / 0.517$ & $0.319 / 0.001^{*}$ & $-0.166 / 0.102$
\end{tabular}

Partial correlation analysis method was employed to calculate the correlation coefficient of the corresponding two variables(eg. TAM in tumor nest and TAM in tumor stroma) with adjusting the other two variables (eg. expression of E-cadherin and expression of Vimentin). ${ }^{*} p<0.05$ 


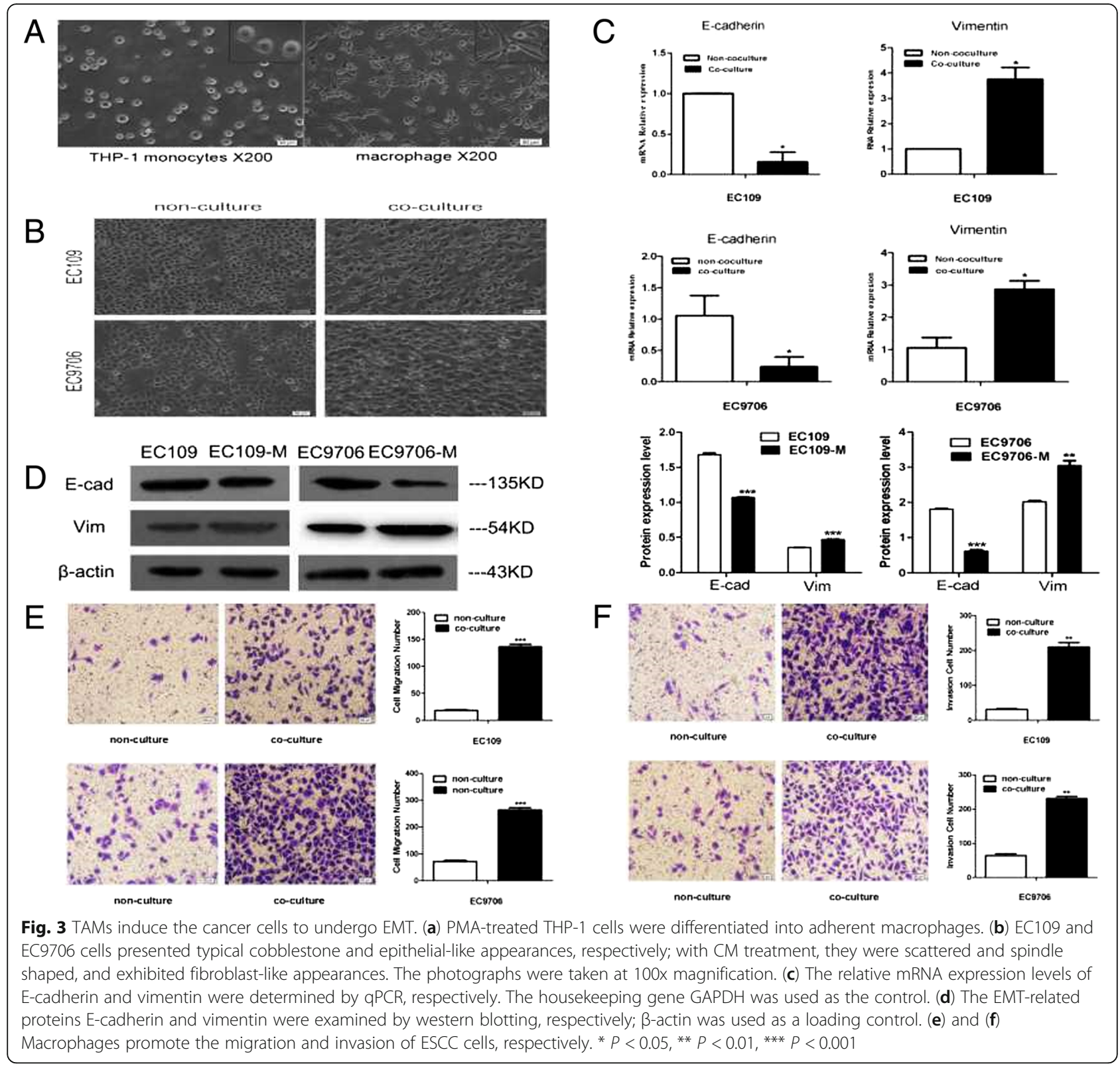

TAMs and transformed into cancer cells with more mesenchymal-like properties [29, 30].

In vitro experiments showed that TAMs not only promote the transformation of ESCC epithelial cells into spindle-shaped mesenchymal cells, but also affect the expression of EMT cell molecular markers (E-cadherin and vimentin) at the mRNA and protein levels. The migration and invasion of ESCC cells were significantly increased, suggesting that TAMs have important biological functions in promoting EMT, invasion, and metastasis of ESCC cells. During this process, TAMs may produce certain cytokines by autocrine and paracrine factors that might play an important role in EMT of ESCC cells. However, the role of these factors was not explored in this present study.
Therefore, in future we plan to further examine the changes in cytokine levels in the co-culture system to explore the specific mechanisms of TAMs in promoting EMT in ESCCs.

\section{Conclusions}

In summary, here, we report the relationship between TAM and EMT in ESCC. TAMs are closely related to cancer cell EMT and are involved in the invasion and metastasis of Kazakh ESCC. Our study emphasizes the importance of the mutual interaction between the TAMs and EMT of cancer cells in tumour progression and provides new ideas for the treatment and prevention of metastasis and recurrence of ESCC. 


\section{Abbreviations}

CANs: Cancer adjacent normal tissues; CM: Conditioned medium; EC: Oesophageal carcinoma; EMT: Epithelial to mesenchymal transition; ESCC: Oesophageal squamous cell carcinoma; IFN-ץ: Interferon gamma; IL10: Interleukin-10; IL-12: Interleukin-12; IL-13: Interleukin-13; IL-4: Interleukin-4; LPS: Lipopolysaccharide; PMA: Phorbol myristate acetate; TAM: Tumourassociated macrophage

\section{Acknowledgments}

Thank Shugang Li for his guidance in statistical analysis, and Yu Ji, Xiaoyue Song for their help in experimental techniques.

\section{Authors' contributions}

LH carried out the experiments, performed the data analysis and writing the manuscript. LCX, ZLY helped with study data collection, interpretation and statistical analysis, LK, JXL, WXL and LWH assisted in preparing the tissue microarray, immunohistochemical staining and scoring all IHC slides. HJM and LF contributed to the design of study and coordination. YL and LKP helped with pathological diagnosis. All the authors contributed to the preparation of the manuscript and approved the final manuscript.

\section{Funding}

This research was supported by grants from the National Natural Science Foundation of China No.81760428, No.81460363, No.81560399, and No.81773116, and National Early Detection and Treatment Project for Upper Digestive Tract in Rural Area in China (NO2018).

\section{Availability of data and materials \\ Data and materials will be shared.}

\section{Ethics approval and consent to participate}

This study was approved by the Medical Ethics Committee of First Affiliated Hospital of Shihezi University Medical College (approval code: 2014-074-01) and the procedures involving human subjects were in accordance with the Declaration of Helsinki and informed consent was obtained from all participants included in the study, in agreement with institutional guidelines.

\section{Consent for publication}

Informed consent for publication was obtained from all participants.

\section{Competing interests}

The authors declare that they have no competing interests.

\section{Author details}

${ }^{1}$ Department of Anatomic and Surgical Pathology, and Key Laboratory of Xinjiang Endemic and Ethnic Diseses, The First Affiliated Hospital, Shihezi University School of Medicine, Xinjiang 832002, People's Republic of China. ${ }^{2}$ Department of Pathology and Medical Research Center, Beijing Chao Yang Hospital, Capital Medical University, Beijing 100020, People's Republic of China. ${ }^{3}$ Department of Pathology, The Sixth Affiliated Hospital of Guangzhou Medical University, Qingyuan People's Hospital, Qingyuan, Guangdong 511518, People's Republic of China. ${ }^{4}$ Department of Neurology, Beijing Friendship Hospital,Capital Medical University, Beijing 100020, People's Republic of China.

Received: 21 December 2018 Accepted: 30 May 2019

Published online: 12 June 2019

\section{References}

1. Ferlay J, Soerjomataram I, Dikshit R, Eser S, Mathers C, Rebelo M, et al. Cancer incidence and mortality worldwide: sources, methods and major patterns in GLOBOCAN 2012. Int J Cancer. 2015;136:359-86.

2. Ayxiam $H, M a H$, llyar $S$, Zhang LW, Ablizi A, Batur M, et al. Metabonomic variation of esophageal cancer within different ethnic groups in Xinjiang, China. Zhonghua Yu Fang Yi Xue Za Zhi. 2009;43:591-6.

3. Zhang BC, Gao J, Wang J, Rao ZG, Wang BC, Gao JF. Tumor-associated macrophages infiltration is associated with peritumoral lymphangiogenesis and poor prognosis in lung adenocarcinoma. Med Oncol. 2011;28:1447-52.

4. Lee CH, Liu SY, Chou KC, Yeh CT, Shiah SG, Huang RY, et al. Tumorassociated macrophages promote oral cancer progression through activation of the Axl signaling pathway. Ann Surg Oncol. 2014;21:1031-7.
5. Lin EW, Karakasheva TA, Hicks PD, Bass AJ, Rustgi AK. The tumor microenvironment in esophageal cancer. Oncogene. 2016;35:5337-49.

6. Quail DF, Joyce JA. Microenvironmental regulation of tumor progression and metastasis. Nat Med. 2013;19:1423-37.

7. Lewis CE, Pollard JW. Distinct role of macrophages in different tumor microenvironments. Cancer Res. 2006;66:605-12.

8. Comito G, Giannoni E, Segura CP, Barcellos-de-Souza P, Raspollini MR, Baroni G, et al. Cancer-associated fibroblasts and M2-polarized macrophages synergize during prostate carcinoma progression. Oncogene. 2014;33:2423-31.

9. Shabo I, Olsson H, Sun HF, Svanvik J. Expression of the macrophage antigen CD163 in rectal cancer cells is associated with early local recurrence and reduced survival time. Int J Cancer. 2009;125:1826-31.

10. Shabo I, Stal O, Olsson H, Dore S, Svanvik J. Breast cancer expression of CD163, a macrophage scavenger receptor, is related to early distant recurrence and reduced patient survival. Int J Cancer. 2008;123:780-6.

11. Lee CH, Espinosa I, Vrijaldenhoven S, Subramanian S, Montgomery KD, Zhu $S$, et al. Prognostic significance of macrophage infiltration in leiomyosarcomas. Clin Cancer Res. 2008;14:1423-30.

12. Yilmaz M, Christofori G. EMT, the cytoskeleton, and cancer cell invasion. Cancer Metastasis Rev. 2009;28:15-33.

13. Klymkowsky MW, Savagner P. Epithelial-mesenchymal transition: a cancer researcher's conceptual friend and foe. Am J Pathol. 2009;174:1588-93.

14. Ansieau S, Courtois-Cox S, Morel AP, Puisieux A. Failsafe program escape and EMT: a deleterious partnership. Semin Cancer Biol. 2011;21:392-6.

15. Su S, Liu Q, Chen J, Chen J, Chen F, He C, et al. A positive feedback loop between mesenchymal-like cancer cells and macrophages is essential to breast cancer metastasis. Cancer Cell. 2014;25:605-20.

16. Jia Z, Zhang Y, Xu Q, Guo W, Guo A. miR-126 suppresses epithelial-tomesenchymal transition and metastasis by targeting PI3K/AKT/snail signaling of lung cancer cells. Oncol Lett. 2018;15:7369-75.

17. Fu XT, Dai Z, Song K, Zhang ZJ, Zhou ZJ, Zhou SL, et al. Macrophagesecreted IL-8 induces epithelial-mesenchymal transition in hepatocellular carcinoma cells by activating the JAK2/STAT3/snail pathway. Int J Oncol. 2015;46:587-96

18. Hu JM, Liu K, Liu JH, Jiang $X L$, Wang $X L$, Chen $Y Z$, et al. CD163 as a marker of M2 macrophage, contribute to predicte aggressiveness and prognosis of Kazakh esophageal squamous cell carcinoma. Oncotarget. 2017;8:21526-38.

19. Hu JM, Liu K, Liu JH, Jiang XL, Wang XL, Yang L, et al. The increased number of tumor-associated macrophage is associated with overexpression of VEGF-C, plays an important role in Kazakh ESCC invasion and metastasis. Exp Mol Pathol. 2017;102:15-21.

20. Hu JM, Chang AM, Chen YZ, Yuan XK, Li F. Regulatory role of miR-203 in occurrence and progression of Kazakh esophageal squamous cell carcinoma. Sci Rep. 2016;6:23780.

21. Park IK, Qian D, Kiel M, Becker MW, Pihalja M, Weissman IL, et al. Bmi-1 is required for maintenance of adult self-renewing haematopoietic stem cells. Nature. 2003;423:302-5.

22. Kubler K, Ayub TH, Weber SK, Zivanovic O, Abramian A, Keyver-Paik MD, et al. Prognostic significance of tumor-associated macrophages in endometrial adenocarcinoma. Gynecol Oncol. 2014;135:176-83.

23. Go Y, Tanaka H, Tokumoto M, Sakurai K, Toyokawa T, Kubo N, et al. Tumorassociated macrophages extend along lymphatic flow in the pre-metastatic lymph nodes of human gastric cancer. Ann Surg Oncol. 2016;23(Suppl 2): 230-5.

24. Hu Y, He MY, Zhu LF, Yang CC, Zhou ML, Wang Q, et al. Tumor-associated macrophages correlate with the clinicopathological features and poor outcomes via inducing epithelial to mesenchymal transition in oral squamous cell carcinoma. J Exp Clin Cancer Res. 2016;35:12.

25. Matejka VM, Finek J, Kralickova M. Epithelial-mesenchymal transition in tumor tissue and its role for metastatic spread of cancer. Klin Onkol. 2017;30:20-7.

26. Park JY, Sung JY, Lee J, Park YK, Kim YW, Kim GY, et al. Polarized CD163+ tumor-associated macrophages are associated with increased angiogenesis and CXCL12 expression in gastric cancer. Clin Res Hepatol Gastroenterol. 2016;40:357-65.

27. Kubota K, Moriyama M, Furukawa S, Rafiul H, Maruse Y, Jinno T, et al. CD163 (+) CD204 (+) tumor-associated macrophages contribute to T cell regulation via interleukin-10 and PD-L1 production in oral squamous cell carcinoma. Sci Rep. 2017:7:1755.

28. Dun EC, Hanley K, Wieser F, Bohman S, Yu J, Taylor RN. Infiltration of tumorassociated macrophages is increased in the epithelial and stromal compartments of endometrial carcinomas. Int J Gynecol Pathol. 2013;32:576-84. 
29. Huysentruyt LC, Mukherjee P, Banerjee D, Shelton LM, Seyfried TN.

Metastatic cancer cells with macrophage properties: evidence from a new murine tumor model. Int J Cancer. 2008;123:73-84.

30. Shabo I, Svanvik J. Expression of macrophage antigens by tumor cells. Adv Exp Med Biol. 2011;714:141-50.

\section{Publisher's Note}

Springer Nature remains neutral with regard to jurisdictional claims in published maps and institutional affiliations.

Ready to submit your research? Choose BMC and benefit from:

- fast, convenient online submission

- thorough peer review by experienced researchers in your field

- rapid publication on acceptance

- support for research data, including large and complex data types

- gold Open Access which fosters wider collaboration and increased citations

- maximum visibility for your research: over $100 \mathrm{M}$ website views per year

At BMC, research is always in progress.

Learn more biomedcentral.com/submissions 